\title{
Epidemiological Description of Dengue Fever Outbreak in Kebridhar District, Somali Region, Ethiopia - 2017
}

\author{
Mikias Alayu ${ }^{1}$, , Fikirte Girma ${ }^{1}$, Mengistu Biru ${ }^{1}$, Tesfalem Teshome ${ }^{2}$, Desalegn Belay ${ }^{1}$ \\ ${ }^{1}$ Public Health Emergency Management Center, Ethiopian Public Health Institute, Addis Ababa, Ethiopia \\ ${ }^{2}$ Department of Public Health, St. Paul Hospital Millennium Medical College, Addis Ababa, Ethiopia
}

Email address:

mikiasalayu7@gmail.com (M. Alayu)

${ }^{*}$ Corresponding author

To cite this article:

Mikias Alayu, Fikirte Girma, Mengistu Biru, Tesfalem Teshome, Desalegn Belay. Epidemiological Description of Dengue Fever Outbreak in Kebridhar District, Somali Region, Ethiopia - 2017. Biomedical Statistics and Informatics. Vol. 4, No. 4, 2019, pp. 27-31.

doi: $10.11648 /$ j.bsi.20190404.11

Received: April 15, 2019; Accepted: May 28, 2019; Published: December 23, 2019

\begin{abstract}
Dengue fever is caused by dengue virus (DENV), a member of the genus Flavivirus, family Flaviviridae. The virus is transmitted by the infected female mosquito called Aedes aegypti. There are four serotypes, DENV1 through DENV4. Dengue fever is one of the most important re-emerging arboviral disease, more than half of the world's population are at risk of this disease. Starting from 2013 over 12,000 cases were reported from Ethiopia. Descriptive cross-sectional study design was applied to describe dengue fever outbreak data from Kebridhar District reported to Ethiopian Public Health Institute from May to June 2017. Ratios, proportions and rates were analyzed by using Microsoft excel and findings were presented by narrations, frequency distributions and graphs. A total of 101 dengue fever cases were reported from Kebridhar District of Somali Region. Sixty-eight-point three percent (69/101) were males and 9.9\% (10/101) cases were hospitalized. The positivity rate of dengue virus was $76.9 \%(10 / 13)$. The median age of cases was 27 years (IQR: $22-38)$. The case fatality rate was zero and the attack rate was 86 cases per 100,000 population. Eighteen-point eight percent (19/101) cases had bleeding. All cases reported that, they had open water containers, no spraying of houses for six months prior to the onset of the fever and bed net utilization rate was $30.7 \%$. Males and $50-54$ years old individuals were highly affected groups. Ministry of Health Regional Health Bureau and District Health Office should work on vector and environmental control activities.
\end{abstract}

Keywords: Dengue Fever, Outbreak, Kebridhar District, Somali Region, Ethiopia

\section{Introduction}

Dengue fever is caused by dengue virus (DENV), a member of the genus Flavivirus, family Flaviviridae. The virus is transmitted by the infected female mosquito called Aedes aegypti. Infections can also be transmitted through blood transfusion, organ transplantation and possibly vertically from mother to child [1]. There are four serotypes of dengue viruses, Dengue virus 1, 2, 3, and 4 [2].

Dengue fever is one of the most important re-emerging arboviral disease worldwide, more than $50 \%$ of the world's population are at risk of this disease and $50 \%$ of these at-risk population were residing in dengue endemic countries of Africa and South East Asia. Around the world, currently over 3.6 billion people are at risk of dengue infection and an estimated of 390 million dengue infections occur worldwide annually [3].

Rapid population growth, unplanned urbanization, and increased international travel in combination with vector distribution were assumed to result in extensive transmission of dengue virus in Africa [4].

Open water storage containers that provide favorable breeding sites for Aedes aegypti, patient's reluctance to travel long distances for medical care and seek for treatment from local traditional healers contributed to the spread of dengue. Human settlements around an irrigation canals also contribute for the spread of dengue fever outbreaks [5].

During the spring and summer seasons the prevalence of dengue virus antibody positivity become higher with a peak at August. Urban and semi urban residence, occupation, the presence of uncovered water container, and lack of mosquito 
net use were the factors associated with positivity of DENV antibody in Ethiopia [6].

Stating from 2013, over 12,000 dengue fever cases were reported from two regions and one city administration of Ethiopia. The first confirmed dengue fever case in Ethiopia was reported in September 2013 from Dire Dawa City. Later on suspected and confirmed cases were reported from Afar Region in 2014 and Somali Region from January 2014 to June 2017 from different districts [7].

Somali Region of Ethiopia experienced different dengue fever outbreaks from 2014 to 2017, the aim of this study was to describe dengue fever outbreak data by person, place and time. This enables to understand the most vulnerable group for similar outbreaks in the future. This study also describes known risk factors of dengue fever.

\section{Methods}

\subsection{Study Area and Period}

The data was collected since $13^{\text {th }}$ May 2017 to $3^{\text {rd }}$ June 2017 from Kebridhar District of Somali Region and compiled at Ethiopian Public Health Institute (EPHI), Public Health Emergency Management Center (cPHEM) database. Kebridhar District is located at $1006 \mathrm{~km}$ away from Addis Ababa, the capital of Ethiopia and $380 \mathrm{~km}$ away from Jigjiga, the capital of Somali Region. The district has a total population of $117,222,57 \%(66,817)$ of them were males, $43 \%(50,405)$ were females and $12(14,067)$ were children below five years of age. The district has two public hospitals.

\subsection{Study Design}

Descriptive cross-Sectional study design was applied to analyze dengue fever outbreak line list data from Kebridhar District of Somali Region.

\subsection{Data Source and Type}

The source of the data for this descriptive analysis was the outbreak data base of Ethiopian Public Health Institute (EPHI), Public Health Emergency Management Center (PHEM). This line list was reported from Kebridhar District of Somali Region.

\subsection{Data Analysis and Presentation}

The data was checked for completeness then cleaned. Ratios, proportions and rates were analyzed by using Microsoft excel 2016. The result was presented by narrations, frequency distributions, bar graphs and histograms.

\subsection{Ethical Considerations}

Written proposal was submitted to Ethiopian Public Health Institute (EPHI), Public Health Emergency Management Center (cPHEM) and permission to analyze the data was obtained.

\section{Result}

A total of 101 dengue fever cases were reported from Kebridhar District starting from $13^{\text {th }}$ May 2017 to $3^{\text {rd }}$ June 2017. The highest number of cases reported per a day are 17, which was on $24^{\text {th }}$ May 2017. The number of cases were increasing from one case on $13^{\text {th }}$ May 2017 to 17 cases on $24^{\text {th }}$ May 2017 and then decreasing to one case on $3^{\text {rd }}$ June 2017 (Figure 1). 90.1\% (91/101) cases were treated as an outpatient and $9.9 \%(10 / 101)$ cases were treated as an inpatient. Thirteen serum samples were collected for confirmation, of those ten were positive for dengue fever sero type two (positivity rate of $76.9 \%(10 / 13)$ ).

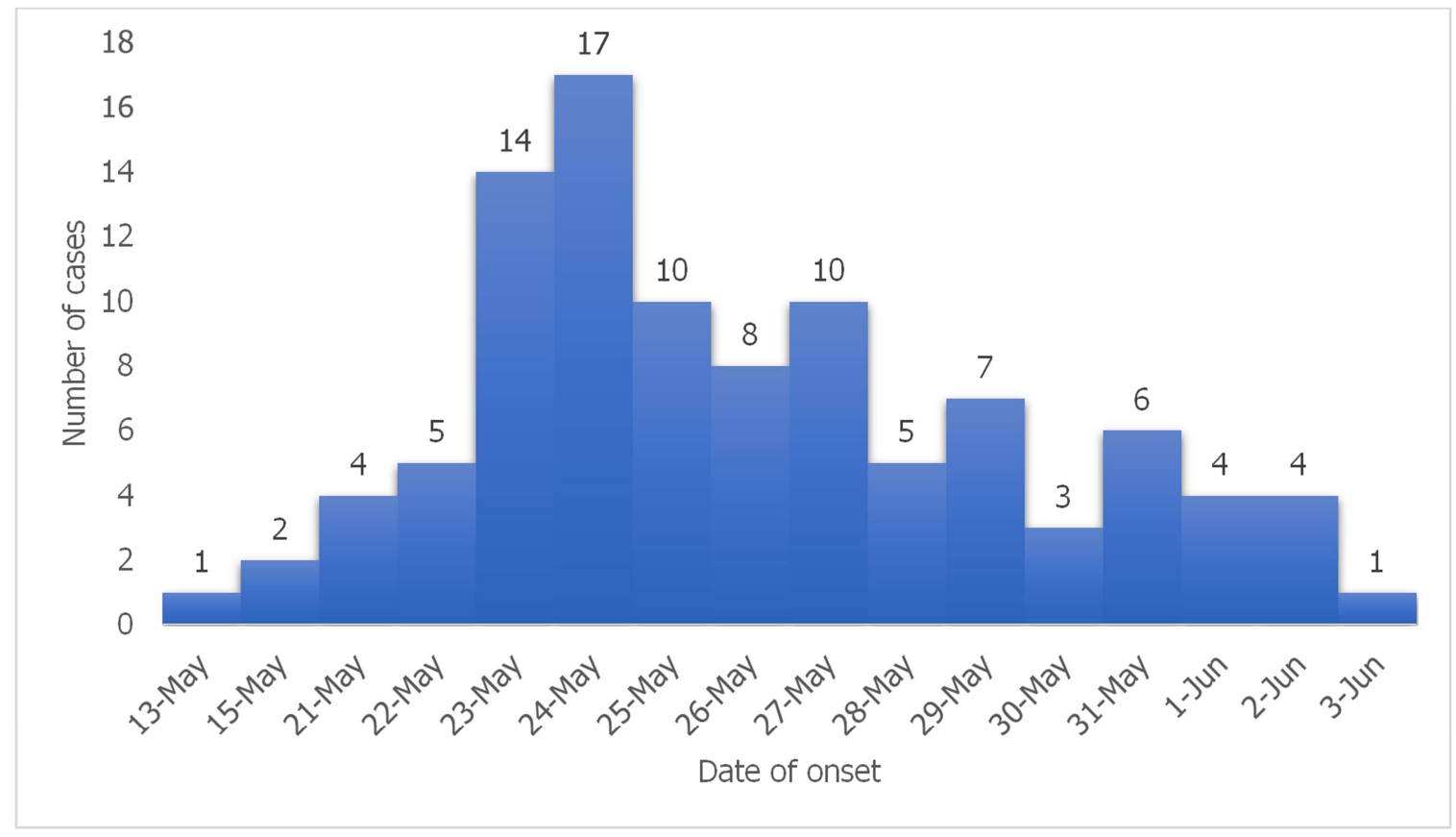

Figure 1. Epidemic curve of dengue fever outbreak in Kebridhar District, Somali Region of Ethiopia. 2017. 
Among a total 101 cases $69(68.3 \%)$ were males and 32 (31.7\%) were females. All cases were above five the age of years old and the highest proportion of cases were between twenty and twenty-four years old $(23.8 \%(24 / 101))$ followed by between twenty-five and twenty-nine years old $(18.8 \%$ $(19 / 101)$ and between fifteen and nineteen years old $(15.8 \%$ $(16 / 101))$ (Table 1). The median age of cases is 27 years (IQR: $22-38)$.

Table 1. Cases distribution by age group and sex, Kebridhar District, Somali Region of Ethiopia, 2017.

\begin{tabular}{|c|c|c|c|c|c|c|c|}
\hline S. $\mathbf{N}$ & Age group & Cases & percentage & Male & Percentage & Female & Percentage \\
\hline 1 & $5-9$ & 1 & $1 \%$ & 1 & $100 \%$ & 0 & $0 \%$ \\
\hline 2 & $10-14$ & 1 & $1 \%$ & 1 & $100 \%$ & 0 & $0 \%$ \\
\hline 3 & $15-19$ & 16 & $15.8 \%$ & 10 & $62.5 \%$ & 6 & $37.5 \%$ \\
\hline 4 & $20-24$ & 24 & $23.8 \%$ & 16 & $66.7 \%$ & 8 & $33.3 \%$ \\
\hline 5 & $25-29$ & 19 & $18.8 \%$ & 14 & $73.7 \%$ & 5 & $26.3 \%$ \\
\hline 6 & $30-34$ & 9 & $8.9 \%$ & 8 & $88.9 \%$ & 1 & $11.1 \%$ \\
\hline 7 & $35-39$ & 8 & $7.9 \%$ & 6 & $75 \%$ & 2 & $25 \%$ \\
\hline 9 & $45-49$ & 4 & $4 \%$ & 1 & $25 \%$ & 3 & $75 \%$ \\
\hline 10 & $50-54$ & 7 & $6.9 \%$ & 4 & $57.1 \%$ & 3 & $42.9 \%$ \\
\hline 11 & $55-59$ & 2 & $2 \%$ & 0 & $0 \%$ & 2 & $100 \%$ \\
\hline 12 & 60 and above & 6 & $5.9 \%$ & 4 & $66.7 \%$ & 2 & $33.3 \%$ \\
\hline Total & & 101 & $100 \%$ & 69 & $68.3 \%$ & 32 & $31.7 \%$ \\
\hline
\end{tabular}

The case fatality rate of this dengue fever outbreak was zero. The overall attack rate of the outbreak was 86 dengue fever case per 100,000 population. The sex specific attack rate for this outbreak were, 103 dengue fever cases per 100,000 male population for males and 63 dengue fever cases per 100,000 female population for females. Individuals between the age of fifty and fifty-four years old were the most affected group with age specific attack rate of 218 dengue fever cases per 100,000 population of the same age group and the least affected age group was children below five years old with zero attack rate (Figure 2).

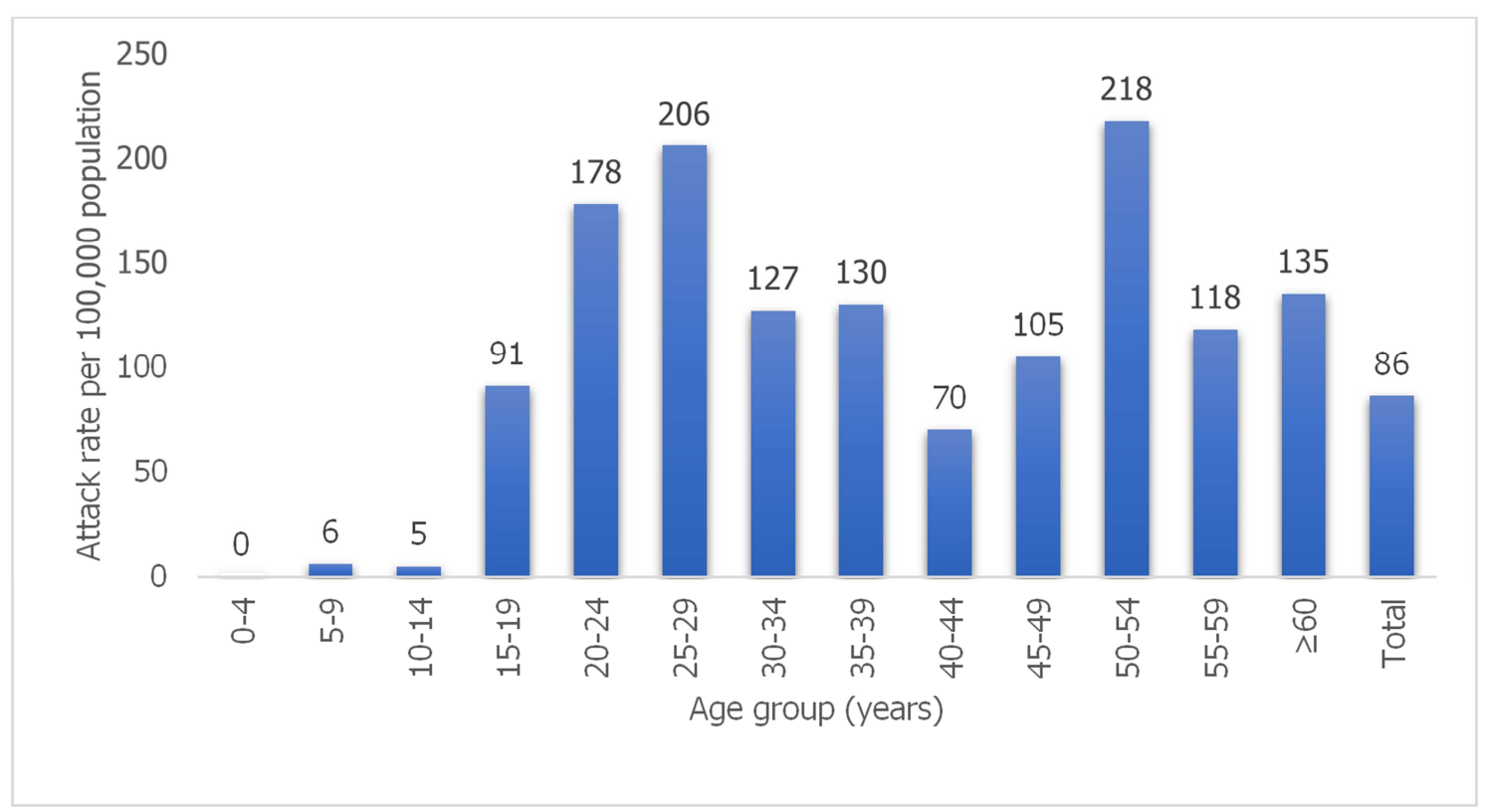

Figure 2. Attack rate of dengue fever outbreak per 100,000 population by age group in Kebridhar District, Somali Region of Ethiopia 2017.

All cases had fever, 92.1\% (93/101) had Headache, 99\% (100/101) had joint pain, 18.8\% (19/101) had nasal bleeding, $49.5 \%$ (50/101) had vomiting and 64.4\% (65/101) cases were presented with back pain (Table 2).

Table 2. Cases distribution by sign and symptoms in Kebridhar District, Somali Region of Ethiopia, 2017.

\begin{tabular}{llllll}
\hline S. N & Sign and symptoms & Yes & Percentage & No & Percentage \\
\hline 1 & Fever & 101 & $100 \%$ & 0 & $0 \%$ \\
2 & Headache & 93 & $92.1 \%$ & 8 & $7.9 \%$ \\
3 & Joint pain & 100 & $99.0 \%$ & 1 & $1.0 \%$ \\
4 & Nasal bleeding & 19 & $18.8 \%$ & 82 & $81.2 \%$ \\
5 & Vomiting & 50 & $49.5 \%$ & 51 & $50.5 \%$ \\
6 & Back pain & 65 & $64.4 \%$ & 36 & 101 \\
\hline
\end{tabular}


Of the possible risk factors of dengue fever, all cases have an open water container in their house, $69.3 \%(70 / 101)$ have never utilize bed net, the house holds of all cases were not yet sprayed since six months prior to the onset of fever and $9.9 \%$ $(10 / 101)$ cases were traveled to dengue fever affected areas with in fourteen days prior to the onset of fever (Table 3).

Table 3. Cases distribution by exposure to the possible risk factors for dengue fever disease, Kebridhar District, Somali Region of Ethiopia, 2017.

\begin{tabular}{|c|c|c|c|c|c|c|}
\hline S. $\mathbf{N}$ & Factors & Yes & Percentage & No & Percentage & Total \\
\hline 1 & Presence of open water container in the house & 101 & $100 \%$ & 0 & $0 \%$ & 101 \\
\hline 2 & Bed net utilization & 31 & $30.7 \%$ & 70 & $69.3 \%$ & 101 \\
\hline 3 & House sprayed in the last six months of the outbreak & 0 & $0 \%$ & 101 & $100 \%$ & 101 \\
\hline 4 & $\begin{array}{l}\text { Travel to affected areas with in fourteen days prior to date } \\
\text { of onset of symptoms }\end{array}$ & 10 & $9.9 \%$ & 91 & $90.1 \%$ & 101 \\
\hline
\end{tabular}

\section{Discussion}

The descriptive analysis of this dengue fever outbreak indicates $68.3 \%$ of cases were male, $9.9 \%$ were hospitalized and the positivity rate was $76.9 \%$. Similarly, another study conducted in Somali Region revealed, the majority of cases $(58 \%)$ were males, the proportion of hospitalized cases were $34 \%$ and the positivity rate was $57.9 \%$ [7]. But the outbreak investigation in Mozambique showed $51 \%$ of cases were females, $30 \%$ of cases were hospitalized and the positivity rate was $23.1 \%$ [8]. An epidemiological and entomological investigation in China showed male to female ratio of cases was 1:0.64 (98/152) and the median age was 34 years [9].

This study shows that median age of cases was 27 years (IQR: 22 - 38) and there were no cases among under five children. The highest proportion of cases were among the age group $20-24$ years old (23.8\%). The Mozambique outbreak investigation result also shows the median age was 28 years (IQR: 18 - 35) [8]. The previous study in Somali Region also showed that, the median age of the cases were 26 years and the highest proportion of cases were among the age group of $15-45$ years old but this study indicated that, $7.7 \%$ of cases were under five years old children [7]. The study in Malaysia also showed the highest proportion of dengue fever cases (38\%) were among the age group 20 - 29 years old [10].

The case fatality rate and attack rate of this outbreak were zero and 86 per 100,000 population respectively. The sex and age specific attack rates were 103 cases per 100,000 male population for males and 63 cases per 100,000 female population for females and zero for under five years children and 208 cases per 100,000 population for $50-54$ years old population. But the study in Guangdong, China revealed that the case fatality rate was 13.3 deaths per 100,000 cases of dengue fever [11]. The other study in India showed, an overall attack rate of 271 per 100,000 population, the highest sex specific attack rate was among females $(299 / 100,000)$ and the highest age specific attack rate was among age group of above 60 years old $(190 / 100,000)$. The case fatality rate in that study was 3 per 1000 dengue fever cases [12].

In this outbreak all cases were manifested with fever and $18.8 \%$ of cases were manifested with bleeding. This finding goes with the finding of the study in dengue fever outbreak investigation in Kenya, where all cases were presented with fever and $13 \%$ of cases were presented with hemorrhage
[13]. Similarly in Mozambique's dengue fever outbreak, 19\% of cases were presented with hemorrhage [8].

This descriptive analysis of dengue fever outbreak data also identified all cases had an open water container in their house and all of them also never sprayed their house holds for six months prior to the outbreak. Bed net utilization rate was $30.7 \%$. This findings were supported by the findings of the study in Sudan which identifies mosquito net utilization and mosquito control practice had a significant association with dengue fever outbreak [1]. The outbreak investigation in North Eastern Kenya also identified that only 9\% of cases reported that, they had indoor and outdoor spraying by health authorities [13].

\section{Conclusion}

This study indicates that the highest proportion of cases were males and among age group of $20-24$ years old. There were no cases among under five years old children. No death was reported in this outbreak and the highest attack rates were among males and among age group of $50-54$ years old. All the cases were manifested with fever, the coverage of indoor and outdoor spray was zero and all cases had an open water container in their houses.

Protective cloths and skin repellants distribution, cover or drain the water containers in and around the residency areas, indoor and outdoor spraying of larvicidal and insecticidal chemicals and community mobilization and engagement are the major preventive mechanisms.

\section{Acknowledgements}

Authors would like to acknowledge Public Health Emergency Management Center (PHEM) of Ethiopian Public Health Institute (EPHI) for providing the data. No fund was provided for this study.

\section{Competing of Interest}

All authors had declared that they had no any competing of interest.

\section{Authors Contributions}

MA designs the study, analysis and write the manuscript. 
FG and MB were worked on data filtering from the PHEM data base and cleaning of the data. DB did laboratory investigation for confirmation of the cases. TT review and comment on the design and the manuscript. Finally, all authors were reviewed and approved the manuscript.

\section{Abbreviations}

DENV, Dengue virus; DHF, Dengue hemorrhagic fever; DSS, Dengue shock syndrome; ELISA, Enzyme-linked immunosorbent assay; IgG, Immunoglobulin G; PCR, Polymerase chain reaction.

\section{References}

[1] Eldigail MH, Adam GK, Babiker RA, Khalid F, Adam IA, Omer $\mathrm{OH}$, et al. Prevalence of dengue fever virus antibodies and associated risk factors among residents of El-Gadarif state, Sudan. BMC Public Health. 2018; 18 (1): 1-8.

[2] Lew RJ, Tsai W, Wang W. Dengue outbreaks in Hawai After WWII - A Review of Public Health Response and Scientific Literature. 2018; 77 (12): 315-8.

[3] Lutomiah J, Barrera R, Makio A, Mutisya J, Koka H, Owaka S, et al. Dengue Outbreak in Mombasa City, Kenya, 20132014: Entomologic Investigations. PLoS Negl Trop Dis. 2016; 10 (10): 2013-4.

[4] Woyessa A. The emergence of dengue fever in Ethiopia. Ethiop J Heal Dev. 2014; 28 (3): 153-4.

[5] Ismail S, Zulkifli M, Mansor R, Mat Yusof M, Ismail MI. Repetitive dengue outbreaks in East Africa: A proposed phased mitigation approach may reduce its impact, Pertanika J Soc Sci Humanit. 2016; 24 (October): 93-100.
[6] Ferede G. Study gauges impact of dengue virus on Ethiopia (2018, May 31) retrieved 21 January 2019 from https://medicalxpress.com/news/2018-05-gauges-impactdengue-virus-ethiopia.html

[7] Ahmed YM, Salah AA. Epidemiology of Dengue Fever in Ethiopian Somali Region: Retrospective Health Facility-based Study. Cent African J Public Heal. 2016; 2 (2): 51-6.

[8] Massangaie M, Pinto G, Padama F, Chambe G, Da Silva M, Mate I, et al. Clinical and epidemiological characterization of the first recognized outbreak of dengue virus-type 2 in Mozambique, 2014. Am J Trop Med Hyg. 2016; 94 (2): $413-$ 6.

[9] Wang J, Chen H, Huang M, Zhang Y, Xie J, Yan Y, et al. Epidemiological and etiological investigation of dengue fever in the Fujian province of China during 2004-2014. Sci China Life Sci. 2017; 60 (1): 72-80.

[10] Jamaiah I, Rohela M, Nissapatorn V, Hiew FT, Mohammad Halizam A, Noor Liani H, et al. Retrospective Study of Dengue Fever (DF) and Dengue Hemorrhagic Fever (DHF) Patients at University Malaya Medical Center, Kuala Lumpur, Malaysia in the Year 2005. Southeast Asian J Trop Med Public Health [Internet]. 2007; 38 (suppl 1): 224.

[11] Li MT, Sun GQ, Yakob L, Zhu HP, Jin Z, Zhang WY. The driving force for 2014 dengue outbreak in guangdong, China. PLoS One. 2016; 11 (11): 1-19.

[12] Kumar S. Dengue Fever in a Municipality of West Bengal, India, 2015: An Outbreak Investigation. Indian J Public Health. 2017; 61: 199-204.

[13] M. O, A. F, V. O. Investigation of laboratory confirmed dengue outbreak in north-eastern Kenya, 2011. PLoS One. 2018; 13 (6): 1-11. 